\title{
The Burden for Caring Patients on Maintenance Hemodialysis is Influenced by Social and Demographic Factors
}

\author{
Rongzhi Zhang ${ }^{1 \#}$, Xiaohui Cui ${ }^{\# \#}$, Haiying Zhuang ${ }^{1}$, Wenjun Xie ${ }^{1}$, Lujie Iv ${ }^{1}$, Yang Liu ${ }^{1}$ and Feng Zheng ${ }^{1,2^{*}}$ \\ ${ }^{1}$ Hemodialysis Center, Department of Nephrology, the Second Hospital, Dalian, China \\ ${ }^{2}$ Advanced Institute for Medical Sciences, Dalian Medical University, Dalian, China
}

"Corresponding author: Feng Zheng, Department of Nephrology, the Second Hospital and Advanced Institute for Medical Sciences, Dalian Medical University,\#9 West, LvShun South Road, Dalian, China, 116044, Tel: 0086-411-86110223; Fax: 0086-411-86110223; E-mail: Zhengfeng@dmu.edu.cn

\#These authors contributed equally.

Rec date: November 04, 2016; Acc date: December 13, 2016; Pub date: December 20, 2016

Copyright: @ 2016 Zhang R, et al. This is an open-access article distributed under the terms of the Creative Commons Attribution License, which permits unrestricted use, distribution, and reproduction in any medium, provided the original author and source are credited.

\begin{abstract}
Caregiving by family members is an invaluable and essential support for chronic ill patients. However, physical and psychological stress associated with long-term caregiving may also affect the well-being and life quality of caregivers. Herein we assessed the burden for caring patients on maintenance hemodialysis by primary family caregivers. 151 patients and their 151 primary family caregivers were recruited. Patients were on hemodialysis for an average of 3.56 years. Self-perceived Zarit Caregiver Burden Scale and WHO-5 well-being index were measured in caregivers. Caregiving related physical and emotional distress was present in 115 out of 151 caregivers. Social and demographic factors including age, educational levels, caring hours and relationship with patients were correlated with the severity of burden. Additionally, caring for patients with diabetes, multiple comorbidities, lower income and higher medical expense increased the burden for caregivers. In conclusion, our study demonstrated that caregiver burden was commonly present in family caregivers of patients on maintenance hemodialysis. Several social and demographic factors in caregivers and patients contributed to caregiver burden.
\end{abstract}

Keywords: Caregiver burden; Hemodialysis; End stage renal disease

\section{Introduction}

Maintenance hemodialysis is the mostly applied renal replacement therapy for patients with end-stage renal disease. Although hemodialysis has become a highly safe medical procedure that significantly extended life of uremic patients, [1-3] it is nevertheless a stressful and lengthy treatment process with few but ever-present minor to severe adverse events such as equipment or facility failures, vascular access dysfunction, needle disconnection and infiltration, blood pressure abnormalities, and dialysis circuit or blood line clotting. Moreover, hemodialysis is usually performed at clinic or hospital where patients are required to attend for a four-hour treatment section three times per week [4]. Since uremic patients are commonly comorbid with various diseases, i.e., frailty, hypertension, diabetes, cardiovascular disease, infection, and mineral and bone disease, maintenance hemodialysis remains problematic and physically and psychologically challenging to patients [5]. Therefore, it is essential to provide consistently both medical and personal life cares for hemodialysis patients.

In China, end-stage renal disease is still considered as a fatal disease to the most of patients and the task of personal life care for patients in maintenance hemodialysis falls primarily on one of the core family members, i.e., spouses, parents, adult-children, siblings, and daughteror son-in-laws. The caregiver is expected to aid in patient's daily life and most of medical activities in between the dialysis [6-8]. When patient becomes more ill or has acute event, new disease(s) and complication(s), caregiver's workloads and responsibilities are overwhelmed, resulting in increased physical, emotional and financial burden and stress [9-11]. Survey on caregivers for hemodialysis patients from other ethnics and countries have revealed the presence of significant physical and psychological stress and burden that affect the quality of life and mental and physical health of caregivers [12-14]. Since traditional Chinese culture would not allow the family caregiver to have an open complain, this may further add the stress levels and psychological burden. In this study, we examined the burden of caregivers from a single Chinese hemodialysis center and explored the factor(s) that affected the burden.

\section{Subjects and Methods}

This survey was conducted on family caregivers of 151 patients at hemodialysis center of the Second Hospital, Dalian Medical University between January and June 2016 (Figure 1). Informed consent was obtained from each patient before the study. Their main caregivers who were over 18 year-old without hearing difficulty or mental disease and agreed to participate were recruited. Both patients and their caregivers were informed that they had right to withdraw from the study at any time without reason.

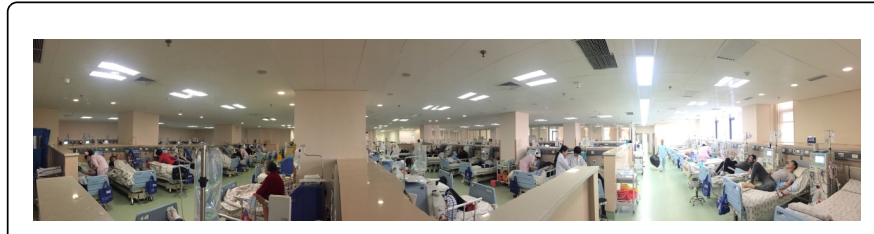

Figure 1: Dialysis center at Second Hospital.

The Zarit Caregiver Burden Scale (ZCBS) is a self-measured 22-item questionnaire with a five-item response set ranging from "never" to "nearly always", a score of 0 to 20 points meaning little or no burden, 
Page 2 of 4

21-40 points meaning mild to moderate burden, 41-60 points meaning moderate to severe burden, and above 61 points meaning severe burden [15]. ZCBS has been used extensively to assess caregiver burden in various chronic diseases and ethnicities and has also been adapted to Chinese with high reliability and validity. Thus, we also applied ZCBS to determine the caregiver burden for hemodialysis patients. The World Health Organization Five-item Well-Being Index (WHO-5) was assessed at the same time with a score ranging from 0 to 25,0 indicating worst possible and 25 indicating best possible quality of life. A score below 13 or an answer of $0-1$ in any of 5 items would be tested for major depression under the International Classification of Diseases. WHO-5 has shown sufficient validity for depression screening and has been applied successfully without modification in Chinese [16,17]. All questionnaires were filled in privately by each caregiver and an independent investigator who has a national certificate as a psychological counsellor was assigned to give instructions and provide assistance on questionnaire related issue(s) raised by caregivers.

\section{Statistical analysis}

Continuous variables were expressed as mean \pm SD. Categorical variables were expressed as number. Since there were uneven distributions of Zarit scores in our caregivers, Log 2 transformation in all values were performed and normal distributions were achieved after the correction. The differences among the variables were analyzed using the independent samples $t$ tests and one-way ANOVA. The Linear Relationship Between two quantitative variables was determined by Pearson correlation analysis. A significance level of 0.05 was used for all tests. All statistical analyses were performed using SPSS19.0 software.

\section{Results}

\section{Demographic characteristics of patients and caregivers}

Among 151 patients, there were 64 females and 87 males. The mean age was $61.8 \pm 11.63$ years old. Patients had been on maintenance hemodialysis for an average of 3.56 years (Table 1). Diabetic nephropathy was the cause of end stage renal disease in 52 patients while 99 patients had other causes such as chronic glomerulonephritis, hypertension and adult polycystic kidney disease (data not shown). The number of patients with less or more than 2 co-morbidities and other demographic characteristics in patients including annul income and monthly medical expense were also listed on (Table 1).

\begin{tabular}{|c|c|c|c|}
\hline & & Patients & Patients (\%) \\
\hline \multirow[t]{2}{*}{ Sex } & Female & 64 & 42 \\
\hline & Male & 87 & 58 \\
\hline \multirow[t]{2}{*}{ Age (years) } & $<65$ & 31 & 21 \\
\hline & $\geq 65$ & 120 & 79 \\
\hline \multirow[t]{2}{*}{ Annual income (¥) } & $<100000$ & 77 & 51 \\
\hline & $\geq 100000$ & 74 & 49 \\
\hline \multirow[t]{2}{*}{ Medical cost (¥/month) } & $<1000$ & 14 & 9 \\
\hline & $1000-1999$ & 37 & 25 \\
\hline
\end{tabular}

\begin{tabular}{|l|l|l|l|}
\hline & $2000-2999$ & 49 & 32 \\
\hline & $>3000$ & 51 & 34 \\
\hline Underlying disease & Diabetes & 52 & 34 \\
\hline & Others & 99 & 66 \\
\hline Comorbidities & $\leq 2$ & 78 & 52 \\
\hline Hemodialysis (years) & $<5$ & 73 & 48 \\
\hline & $\geq 5$ & 109 & 72 \\
\hline
\end{tabular}

Table 1: Social and demographic characteristics of patients.

Among 151 primary caregivers, there were 97 females and 54 males. The mean age was $54 \pm 11.63$ years old. 141 (93\%) caregivers had relationship with patients as spouses, parents, or adult children. The rest of caregivers $(n=10,7 \%)$ were mostly siblings and daughter and son-in-laws. 117 out of 151 (77\%) caregivers did full-time care for patients and were without or had retired or quitted the job. As shown on (Table 2), 82 caregivers spent $12-24 \mathrm{~h}$ a day for care-related activities. However, there were also 38 individuals who spent less than $4 \mathrm{~h}$ a day for caregiving.

\begin{tabular}{|c|c|c|c|}
\hline & & Caregivers & Caregivers (\%) \\
\hline \multirow[t]{2}{*}{ Sex } & Female & 97 & 64 \\
\hline & Male & 54 & 36 \\
\hline \multirow[t]{2}{*}{ Age (Years) } & $<65$ & 53 & 35 \\
\hline & $\geq 65$ & 98 & 65 \\
\hline \multirow[t]{3}{*}{ Marital status } & Single & 10 & 6.6 \\
\hline & Married & 137 & 90.7 \\
\hline & Widow & 4 & 2.6 \\
\hline \multirow[t]{2}{*}{ Job } & No & 116 & 77 \\
\hline & Yes & 35 & 23 \\
\hline \multirow[t]{3}{*}{ Education } & Primary school & 11 & 7.3 \\
\hline & High school & 40 & 26.5 \\
\hline & College & 100 & 66.2 \\
\hline \multirow[t]{4}{*}{ Time (Hours/day) } & $0-3.9$ & 38 & 25 \\
\hline & $4-7.9$ & 13 & 8.6 \\
\hline & $8-11.9$ & 18 & 11.9 \\
\hline & $>12$ & 82 & 54.3 \\
\hline \multirow[t]{2}{*}{$\begin{array}{l}\text { Caregiver-patient } \\
\text { relationship }\end{array}$} & $\begin{array}{l}\text { Spouse/Parent } \\
\text { /Adult child }\end{array}$ & 141 & 93 \\
\hline & Other relatives & 10 & 7 \\
\hline
\end{tabular}

Table 2: Social and demographic characteristics of caregivers. 
Page 3 of 4

\section{Caregiver burden}

Zarit score system ZCBS and WHO-5 were used in 151 primary caregivers for hemodialysis patients to assess their quality of life and the status of physical and mental health.

77 out of 151 caregivers (51\%) reported mild to moderate burden. Moderate to severe burden was reported in 38 caregivers (25.2\%). Age was a contributing factor to care burden. Pearson analysis revealed that there was a moderate correlation between caregiver age and care burden $(\mathrm{r}=0.235, \mathrm{p}<0.01$, Table 3$)$.

\begin{tabular}{|l|l|l|}
\hline Caregivers & ZCBS $(\log 2)$ & $\mathbf{r}, \mathbf{p}$ value \\
\hline Age: $54 \pm 11.6$ & $4.95 \pm 0.61$ & $r=0.235, p=0.01$ \\
\hline WHO-5: $14.5 \pm 6.5$ & & $r=0.596, p=0.001$ \\
\hline
\end{tabular}

The correlation of age and self-perceived well-being, as measured by WHO-5 index with caregiver burden was tested by Pearson's correlation analysis. ZCBS scores were transformed to log 2 value for normal distribution.

Table 3: Age, self-perceived well-being and caregiver burden.

The educational level of caregiver was also a factor in influencing the degree of burden. The higher educational levels of caregivers as defined by the above college graduate were associated with lesser burden (ZCBS scores, $4.74 \pm 0.58$; vs. $5.09 \pm 0.59$ in caregivers with lower education levels, $\mathrm{p}=0.000$, Table 4 ). Moreover, the relationship between caregiver and patient played a significant role in affecting the levels of burden. Spouses, parents and adult children felt more stressful and burdened than other caregivers. Additionally, the self-perceived well-being as determined by WHO- 5 well-being index was inversely correlated with the score of ZCBS, namely the better WHO-5 score, which suggests a better quality of life was associated with the lesser burden $(r=-0.596, p<0.01$, Table 3$)$. Similarly, caregivers who still had job or spent less than $4 \mathrm{~h}$ per day in caregiving activities had less selfperceived burden than those who were without job or spent more than $12 \mathrm{~h}$ per day in caregiving (Table 4 ).

\begin{tabular}{|l|l|l|l|l|l|}
\hline & Factors & & Case (n) & $\begin{array}{l}\text { ZCBS (log } \\
\text { 2) }\end{array}$ & P value \\
\hline Caregivers & Job & Yes & 116 & $5.03 \pm 0.18$ & 0.002 \\
\hline & No & 35 & $4.67 \pm 0.61$ & \\
\hline & Education & Low & 92 & $5.09 \pm 0.59$ & 0.000 \\
\hline & $\begin{array}{l}\text { Time (hours/ } \\
\text { day) }\end{array}$ & $<4$ & 38 & $4.70 \pm 0.57$ & 0.048 \\
\hline & High & 59 & $4.74 \pm 0.58$ & \\
\hline & $4-7.9$ & 13 & $4.91 \pm 0.68$ & \\
\hline & $8-11.9$ & 18 & $4.94 \pm 0.69$ & \\
\hline Patients & $\begin{array}{l}\text { Medical cost } \\
\text { (¥/month) }\end{array}$ & $\leq 1000$ & 14 & $4.56 \pm 0.36$ & 0.044 \\
\hline & $\begin{array}{l}\text { Annual } \\
\text { Income (¥) }\end{array}$ & $\leq 100000$ & 77 & $5.08 \pm 0.58$ & 0.01 \\
\hline & $>100000$ & 74 & $4.82 \pm 0.61$ & \\
\hline
\end{tabular}

\begin{tabular}{|l|l|l|l|l|l|}
\hline & $1000-1999$ & 37 & $5.04 \pm 0.56$ & \\
\hline & $2000-2999$ & 49 & $4.91 \pm 0.63$ & \\
\hline & $>3000$ & 51 & $5.05 \pm 0.64$ & \\
\hline & $\begin{array}{l}\text { Underlying } \\
\text { disease }\end{array}$ & Diabetes & 52 & $5.12 \pm 0.60$ & 0.015 \\
\hline & $\begin{array}{l}\text { Comorbiditie } \\
\text { S }\end{array}$ & $\leq 2$ & 78 & $4.93 \pm 0.58$ & 0.02 \\
\hline & Others & 99 & $4.87 \pm 0.60$ & \\
\hline & $\begin{array}{l}\text { Hemodialysis } \\
\text { (Years) }\end{array}$ & $<5$ & 109 & $4.95 \pm 0.61$ & 0.844 \\
\hline & $>2$ & 73 & $5.20 \pm 0.63$ & \\
\hline
\end{tabular}

Table 4: Social and demographic factors contribute to caregiver burden.

From patients' part, patients with underlying disease of diabetes gave their caregivers more burden than patients with other underlying diseases (Table 4). One reason might be that patients with diabetes had more co-morbidities and might need more assistance in personal life care. Indeed, the caregiver burden was significantly increased in patients with more than two co-morbidities. Patient's income levels and medical expense also had significant impact on caregiver burden. Less caregiver burden was found on patients with high annual income (more than 100 thousands yuan) compared to patients with low annual income. Similarly, the amount of medical expense was positively correlated with the levels of caregiver burden. The burden in caregivers for patients who paid less than one thousand yuan a month in medical expense was lower than the burden in caregivers for patients who paid more $(\mathrm{p}<0.05)$.

\section{Discussion}

It is well recognized now that care for patients with chronic fatal illness by family members has two-opposite impacts on physical and mental health of caregivers. One is positive because the obligation and responsibility to take care the loved-one who may loss in any time would give the caregiver all the inspiration to put out the whole efforts. However, long-term caregiving also have a significantly negative influence [18-21]. The physical, emotional, economical and timing strains may overwhelm the caregivers.

End stage renal disease is still considered as a chronic fatal disease although renal replacement therapy including hemo and peritoneal dialysis has helped to prolong the life and improve the quality of life. About $50 \%$ of end stage renal disease patients lost their life after 5 years in hemodialysis in China. Thus, there is always an impending life threatening burden for patients and their caregivers on maintenance hemodialysis. Riou et al., reported that there were $25 \%$ of caregivers feeling stressed and $28 \%$ of caregivers feeling depressed when they cared for Canadian nocturnal home hemodialysis patients [22]. Caregiving burden was also perceived by patients from the Frequent Hemodialysis Network and by caregivers themselves from a dialysis center in Turkey [23-25]. In this study, 51\% of caregivers had mild to moderate and $25.2 \%$ of caregivers had moderate to severe selfperceived burden, suggesting a relatively widespread presence of physical and psychological distress in family caregivers for Chinese 
patients on maintenance hemodialysis. We have further identified factors in caregivers and patients that might contribute to caregiver burden. We found a linear correlation between age and caregiver burden. Moreover, caregivers who needed to spend more time on caring related activities had increased stress and burden. Similarly, full time caregivers had more burden compared to those who still had a job and did care in part time. The education levels in caregivers also seemed to play a role. Caregivers with college or higher education had less self-perceived burden compared to those with high school or lower education. As expected, the status of physical and mental well-being was negatively correlated with caregiver burden. In term of patient and caregiver relationship, we found that parents, spouses and adult children had higher caregiver burden than other family members. Our results are not in completely agreement with the data from Mollaoglu et al., however. They showed that younger age in caregiver was associated with higher burden and spouses as caregiver had lower burden compared to other family members [25]. These discrepancies in self-perceived caregiver burden between our caregivers and theirs may be caused by culture differences. In addition, since both our study and theirs had a relative small number of subjects, a further increase in research subjects is required to confirm the findings and clarify the differences.

We have also identified disease and social and demographic factors in patients that were related to caregiver burden. For instance, patient's underlying diseases, co-morbidities, annual income and medical expense had significant impact on caregiver burden. Patients with diabetes and more co-morbidity caused higher burden in caregivers. Understandably, lower income and more medical expense were associated with higher burden. These social and economic factors have also been shown to contribute to depression and caregiver burden in patients on maintenance hemodialysis and their family caregivers by other group [26].

In conclusions, we reported for the first time the presence of a relative high physical and mental burden in family caregivers of Chinese patients on maintenance hemodialysis. Factors in caregivers such as the age, education level, with or without job, caring hours, patient and caregiver relationship and the status of physical and mental well-being were identified to contribute to caregiver burden. Patient's underlying disease, number of co-morbidities, and income and medical expense also played a role.

\section{References}

1. Denhaerynck K, Manhaeve D, Dobbels F, Garzoni D, Nolte C, et al. (2007) Prevalence and consequences of nonadherence to haemodialysis regimens. Am J Crit Care 16: 222-235.

2. Gayomali C, Sutherland S, Finkelstein FO (2008) The challenge for the caregiver of the patient with chronic kidney disease. Nephrol Dial Transplant 23: 3749-3751.

3. Mollaoglu M (2006) Perceived social support, anxiety and selfcare among patients receiving hemodialysis. Dial Transplant 35: 144-152.

4. LowJ, Smith G, Burns A, Jones J ( 2008) The impact of end-stage kidney disease (ESKD) on close persons: A literature review. NDT Plus 1: 67-79.

5. Einollahi B, Taheri S, Nemati E, Abbaszadeh S, Pourfazadeh S, et al. (2009) Burden among care-givers of kidney transplant recipients and its associated factors. Saudi J Kidney Dis Transpl 20: 30-34.
6. Avsar U, Avsar UZ, Cansever Z, Yucel A, Cankaya E, et al. (2015) Caregiver Burden, Anxiety, Depression, and Sleep Quality Differences in Caregivers of Hemodialysis Patients Compared With Renal Transplant Patients. Transplant Proc 47: 1388-1391.

7. Cohen LM and Germain MJ (2014) Caregiver burden and hemodialysis. Clin J Am Soc Nephrol 9: 840-842.

8. Akkus Y (2011) Multiple sclerosis patient caregivers: the relationship between their psychological and social needs and burden levels. Disabil Rehabil. 33: 326-333.

9. Da Silva-Gene M, Wellsted D, Greenshields H, Norton S, Chandna SM, et al.(2012) Quality of life and survival of patients with advanced kidney failure managed conservatively or by dialysis.Clin J Am Soc Nephrol 7: 2002-2009.

10. Kimmel PL, Peterson RA (2005) Depression in end-stage renal disease patients treated with hemodialysis: Tools, correlates, outcomes, and needs. Semin Dial18: 91-97.

11. Boutoleau-Bretonniere C, Vercelletto M, Volteau C (2008) Zarit burden inventory and activities of daily living in the behavioral variant of frontotemporal dementia. Dement Geriatr Cogn Disord 25: 272-277.

12. Magda M (2014) Bayoumi subjective burden on family carers of hemodialysis patients. Open J Nephrol 4: 79-85.

13. Belasco A, Barbosa D, Bettencourt AR, Diccini S, Sesso R (2006) Quality of life of family caregivers of elderly patients on hemodialysis and peritoneal dialysis. Am J Kidney Dis. 48: 955-963.

14. Shimoyama S, Hirakawa O, Yahiro K, Mizumachi T, Schreiner A, et al. (2003) Health-related quality of life and caregiver burden among peritoneal dialysis patients and their family caregivers in Japan. Perit Dial Int 23: S200-S205.

15. Zarit SH, Reever KE, Back-Peterson J (1980) Relatives of the impaired elderly: Correlates of feelings of burden. Gerontologist 20: 649-655.

16. Ren J, Hong X, Zhao WG, Duan YP, Xiong NN, et al. (2015) Validity and reliability of the Problem Area in Diabetes Scale in patients with type 2 diabetes. Chinese Mental Health Journal 11: 806-811

17. Ou A, Hao YT, Liang ZH (2009) Study on the Mental Health Index Questionnaire for Elder people. Chinese Journal of Health Statistics. 2: 128-130.

18. Retrum JH , Nowels CT , Bekelman DB (2013) Patient and caregiver congruence: the importance of dyads in heart failure care. J Cardiovasc Nurs 28: 129-136.

19. Alnazly EK (2016) Burden and coping strategies among Jordanian caregivers of patients undergoing hemodialysis. Hemodialysis International 20: 84-93.

20. Schulz R, Sherwood PR (2008) Physical and mental health effects of family caregiving. Am J Nurs 108: 23-27.

21. Fisher L (2006) Research on the family and chronic disease among adults: major trends and directions. Fam Syst Health 24: 373 Y380.

22. Rioux, JP, Narayanan R, Chan CT (2012) Caregiver burden among nocturnal home hemodialysis patients. Hemodial Int 16: 214-219.

23. Cantekin I, Kavurmaci M, Tan M (2016) An analysis of caregiver burden of patients with hemodialysis and peritoneal dialysis. Hemodialysis International 20: 94-97.

24. Van Eps CL, Jeffries JK, Johnson DW, Campbell SB, Isbel NM, et al. (2010) Quality of life and alternate nightly nocturnal home hemodialysis. Hemodial Int 14: 29-38.

25. Mollaoglu M, Kayatas M, Yurugen B (2013) Effects on caregiver burden of education related to home care in patients undergoing hemodialysis. Hemodial Int 17: 413-420.

26. Sezer S, Uyar ME, Bal Z, Tutal E, Ozdemir Acar FN (2013) The influence of socioeconomic factors on depression in maintenance hemodialysis patients and their caregivers. Clin Nephrol 80: 342-348. 